\title{
Competencia digital y desarrollo profesional de los docentes de dos instituciones de educación básica regular del distrito de Los Olivos, Lima-Perú
}

\section{Digital competence and professional development of teachers from two institutions of regular basic education of Los Olivos district, Lima-Peru}

\author{
Felipe Guizado Osco ${ }^{1}$ \\ https://orcid.org/0000-0003-3765-7391 \\ Isabel Menacho Vargas ${ }^{2}$ \\ https://orcid.org/0000-0001-6246-4618 \\ Angel Salvatierra Melgar ${ }^{3}$ \\ https://orcid.org/0000-0003-2817-630X \\ Universidad César Vallejo, Perú
}

Vol. 6(1). Enero-abril. Hamut'ay 2019. Lima-Perú

Recibido: $30-10-2018$

Aceptado: 07-04-2019

\section{Cita Recomendada}

Guizado, F., Menacho, I. \& Salvatierra, A. (2019). Competencia digital y desarrollo profesional de los docentes de dos instituciones de educación básica regular del distrito de Los Olivos, Lima-Perú. Hamut'ay, 6(1), 54-70.

http://dx.doi.org/10.21503/hamu.v6i1.1574

\section{RESUMEN}

El avance y desarrollo vertiginoso de la ciencia y el conocimiento que se evidencia en la evolución de las tecnologías constituye, en estos tiempos, una exigencia al cambio en el perfil del profesional de la educación, quien debe desarrollar las competencias digitales idóneas para implementarlas en su proceso de enseñanza aprendizaje. El objetivo fue determinar la relación entre la competencia digital y el desarrollo profesional de los docentes de Educación Básica Regular. El estudio fue de tipo básico, diseño no experimental, correlacional causal y transversal. La selección de la muestra se realizó en dos instituciones educativas pertenecientes a la Red 08 de la Unidad de Gestión Educativa Local No. 02, en Lima, Perú, ( $\mathrm{n}=100$ docentes). Para la recolección de datos se utilizó dos cuestionarios con escala Likert, cuya validez de contenido fue por juicio de expertos, con un nivel de concordancia superior al 0.75 , y la confiabilidad tuvo un índice de 0.77 y 0.75 . En los resultados se concluye que existe correlación entre las competencias digitales docente y el desarrollo profesional determinado por el Chi cuadrado X2c=18.499 con un 1 grado de libertad y el valor de significación estadística de $p=0.00$ a un nivel de confianza del $95 \%$. Y también se obtuvo que el $24 \%$ del desarrollo profesional es explicado por las competencias digitales de los docentes y el $76 \%$ se debe a otros factores.

1 Licenciado en Educación; Magister en Educación con mención Docencia Universitaria, Doctor en Administración de la Educación, Estudios de Posgrado en la Universidad de Buenos Aires y Universidad Complutense de Madrid, Docente a nivel de pregrado y posgrado. E-mail: fguizadoo@ucv.edu.pe 2 Psicóloga, Educadora, Magister en evaluación y Acreditación de la calidad educativa, Doctora en Educación y Doctora en Gestión Pública y Gobernabilidad. Jefe de Investigación de la Escuela de Posgrado en la Universidad César Vallejo. Investigador por Concytec. E-mail: Regina:16036. imenacho@ucv.edu.pe

3 Licenciado en Matemática, Magister en Tecnologías de la Información, Doctor en Ciencias de la Educación por la Universidad Nacional del Centro del Perú, con estudios de Matemática Estadística por la Universidad Nacional Mayor de San Marcos, diplomatura en: Estadística Aplicada por la Pontificia Universidad Católica del Perú.. docente a nivel de pregrado y posgrado en universidades públicas y privadas. E-mail: smelgara@ucv.edu.pe. 
Palabras Clave: Competencia digital, desarrollo profesional, docente.

\begin{abstract}
The progress and accelerated development of science and knowledge that is evident in the evolution of technologies constitute a demand to change the profile of professionals in the education field. Nowadays, they must develop suitable digital skills to implement them in their teaching-learning process. The objective was to determine the relationship between digital competence and the professional development of teachers working in Regular Basic Education. This was a basic study with a causal cross-sectional correlational non-experimental design. The selection of the sample was carried out in two educational institutions belonging to Network 08 of the Local Educational Management Unit No. 02, in Lima, Peru, ( $\mathrm{n}=100$ teachers). For data collection, two Likert-scale questionnaires were used, whose content validity was obtained by expert judgment, with a level of agreement higher than 0.75 , and its reliability had an index of 0.77 and 0.75 . In the results it is concluded that there is a correlation between the digital competences of teachers and their professional development determined by the Chi square X2c $=18.499$ with 1 degree of freedom and the value of statistical significance of $p=0.00$ at a confidence level of $95 \%$. And it was also obtained that $24 \%$ of the professional development is explained by the digital competences of the teachers while $76 \%$ is due to other factors..
\end{abstract}

Keywords: Digital competence, professional development, teacher.

\section{INTRODUCCIÓN}

El que los docentes desarrollen competencias digitales en su ámbito profesional se ha hecho una necesidad impostergable, debido a los cambios de perfiles de sus estudiantes, a quienes imparten catedra, ya que estos son nativos digitales y están inmersos en estos medios dentro y fuera del aula en cada momento de su vida, por lo que el docente de cualquier nivel educativo no puede estar exento de adquirir y desarrollar estas competencias, como también lo enfatiza Durán, Prendes \& Gutiérrez (2019) al referir que la competencia digital es clave en el perfil profesional docente.

Estudios realizados en Latinoamérica también refieren sobre estos aspectos como es el caso de Osorio (2016) en la investigación realizada en educación básica primaria sobre el desarrollo profesional docente en Colombia; concluye que muchos profesores necesitan apoyo y orientación para ser capaces de enseñar programas de innovación educativa y aplicación de las tecnologías. Por su parte el Ministerio de Educación Nacional de Colombia (2013) sobre el estudio realizado de Competencias TIC para el desarrollo profesional docente, enfatiza el fomento hacia la innovación $y$ en la posibilidad de transformar las practicas educativas con la integración pertinente de las TIC, las cuales consideran como herramientas de gestión del conocimiento y facilitadoras de la comunicación global, las cuales tienen un rol importante en el quehacer educativo, (UNESCO, 2010).

Caudillo (2016) analiza desde otra perspectiva el que el docente deba estar capacitado en el uso y apropiación de las tecnologías como parte de su competencia digital, al resaltar que los estudiantes de educación secundaria en Sonora México siguen mostrando una tendencia confirmatoria con el uso autónomo de las tecnologías, lo cual les 
Competencia digital y desarrollo profesional de los docentes de dos instituciones de educación básica regular del distrito de Los Olivos, Lima-Perú

permiten realizar ciertos logros académicos como una búsqueda y una selección de información hasta cierto punto aceptable. Pero este uso de las tecnologías de la información y comunicación no se observa de la misma manera en los docentes, como lo asevera Colás, De Pablo \& Ballesta (2018) al mencionar que existe una insuficiente preparación del docente para introducir en sus sesiones de aprendizaje la aplicación de las tecnologías, como el uso de Internet y de herramientas informáticas.

En el caso de Perú a partir del 2017 con la implementación del cambio en las competencias del Currículo Nacional de Educación Básica, se mira desde otra perspectiva los aspectos relacionados a las competencias digitales docentes. Vargas-D 'Uniam, Chumpitaz-Campos, Suárez-Díaz \& Badia (2014) en su estudio sobre la relación entre las competencias digitales de docentes de educación básica y el uso educativo de las tecnologías en las aulas, concluyen que se identificaron dos tipos de competencias, las competencias tecnológicas, las cuales comprenden el uso de aplicaciones informáticas, como el procesador de texto, hoja de cálculo y base de datos, aplicaciones y presentaciones multimedia, el software educativo y el uso del Internet para comunicación y navegación; en cuanto a la competencia pedagógica es la que incluye las habilidades en el uso y aplicación de las tecnologías en el proceso de enseñanza aprendizaje. Asimismo, mencionan respecto a las competencias digitales que hay un mayor desarrollo en utilizar las tecnologías para actividades sencillas, al realizar una presentación o localizar recursos útiles para sus asignaturas. En cuanto a las competencias tecnológicas consideran que tienen capacidad para diseñar o modificar paginas web o blog, pero que los docentes hacen un escaso uso de las tecnologías en el aula, por lo que concluyeron que existe una correlación no significativa entre las competencias digitales de los docentes y los usos educativos que realizan en el aula, demostrando los resultados un bajo nivel competencial.

Los autores citados nos orientan a hurgar en la brecha entre las competencias digitales de los docentes y estudiantes la cual presenta un reto para ellos, porque en pleno siglo XXI donde el auge de la innovación educativa ha ido en aumento, toda- vía la labor y la idoneidad en el desempeńo de los docentes en apropiarse de las tecnologías es incipiente, situación que se tiene como evidencia en los distintos resultados que arrojan las múltiples evaluaciones de desempeño académico realizadas a estos; ya sea para los ascensos en los distintos niveles como para alcanzar el ingreso a la carrera pública magisterial, (Minedu, 2016). Aunque en menor porcentaje existen instituciones educativas que cuentan con personal docente que tienen altos niveles de desempeño en las distintas áreas que engloban su perfil, y en las que se incluye de manera eficiente y competente el uso y apropiación de las tecnologías, según la evaluación de desempeño realizada por el Minedu (2017).

Por otra parte, el Proyecto Educativo Nacional de educación al 2021, el cual es un referente en el ámbito educativo, destaca que se debe contar con docentes altamente capacitados, con una educación continua y permanente a la par de los avances de las tecnologías, la ciencia y la pedagogía, (CNE, 2006). Al respecto Colomer et al., (2018) refieren que la sociedad en general en estos últimos años ha sufrido una transformación por la influencia de las tecnologías, de la cual no esta exenta la educación, por lo que esta debe estar en sintonía con estos cambios, por ende es cada vez más necesaria la formación y desarrollo profesional de un profesorado que sea competente digitalmente, con capacidad para aprovechar la tecnología ya que el contexto global presenta un avance vertiginoso en el desarrollo de las herramientas de información y comunicación y el conocimiento, en este contexto están incluidos los procesos educativos, los campos educacionales y los distintos espacios donde se promueve el desarrollo de la ciencia.

Es por ello que se presenta la necesidad de realizar una indagación respecto a la competencia digital de los docentes, dentro del contexto de su formación profesional en nuestro país; por lo que en este estudio el objetivo fue determinar la relación entre la competencia digital y el desarrollo profesional de los docentes de Educación Básica Regular, para lo cual se formuló la siguiente hipótesis: existe relación de las competencias digitales y el desarrollo profesional docente y sus dimensiones. 


\section{Competencia digital}

La comunidad europea formula la competencia digital, como una de las competencias básicas y fundamentales, definiéndola como:

El uso crítico y seguro de las tecnologías de la información para el trabajo, el tiempo libre y la comunicación. Apoyándose en habilidades [en el uso] de las Tecnologías de la Información y Comunicación (TIC) básicas: uso de ordenadores para recuperar, evaluar, almacenar, producir, presentar e intercambiar información, y para comunicar y participar en redes de colaboración a través de internet (MECD, 2017, p. 8)

Para el Ministerio de Educación de España (2017) la competencia digital puede definirse "como el uso creativo, crítico y seguro de las tecnologías de información y comunicación para alcanzar los objetivos relacionados con el trabajo, la empleabilidad, el aprendizaje, el tiempo libre, la inclusión y participación en la sociedad" (p.9) así como a la integración sociocultural en un grupo humano digital e imbuido de tecnología. Para Almerich, et al., (2018) las competencias digitales son el conjunto de conocimientos y habilidades que permiten dominar distintos recursos tecnológicos. Desde otra óptica Valdivieso-Guerrero (2013) refiere que es la integración de indicadores de desempeños donde están incluidas las competencias genéricas relacionadas a competencias interpersonales, instrumentales e informacionales y las competencias especificas vinculadas a lo pedagógico-tecnológico y a lo pedagógico-didáctica-disciplinares.

\section{Competencia digital docente}

Un acercamiento a la definición de competencia digital docente lo formulan Zavala et al., (2016, p. 334) al señalar que esta se sustenta en base a su uso de manera creativa, crítica y segura de las tecnologías de información y comunicación como una herramienta para los docentes que les permita llegar a adquirirla como parte de su desarrollo profesional, aprendizaje, entretenimiento, comunicación e información con la sociedad, en el contexto de la escuela. Es esto lo que se pretende develar en el escenario planteado para la localidad en estudio.
La formación profesional docente, por ende, es apostar por asumir la relevancia de la competencia digital bajo el entendimiento de lo que supone ser un docente competente en las diversas esferas de lo educativo; hacer uso de las tecnologías considerando la importancia de saber cómo usar, analizar y reflexionar sobre estas dentro del proceso de enseñanza-aprendizaje, como lo refieren Prendes, Gutiérrez \& Martínez (2018) lo cual hace que tengan un involucramiento pedagógico y social con sus estudiantes.

$\mathrm{Al}$ respecto, Esteve (2015) sostiene que una sociedad globalizada denota cambios a gran velocidad, dentro del marco de la transformación espacio temporal, con una enorme saturación de información, con formas distintas de inteligencia colectiva y en un espacio casi abstracto denominado red, eso supone asumir nuevos retos, que son los desafíos que la educación y las instituciones tienen y están en la necesidad de asumir, dentro del cual el docente es un elemento esencial. En este mismo sentido y expresando la importancia de la competencia digital refieren Sámano et al. (2018) que existe la necesidad de hacer un rediseño curricular con un cambio de fondo en la enseñanza, a la cual se debe incorporar el uso de las tecnologías, haciendo hincapié en la necesidad de plantear la alfabetización digital, como un aprendizaje múltiple, global e integrado de las distintas formas y lenguajes que en la actualidad son importantes en la formación de los docentes, lo cual revertirá en sus estudiantes. Del mismo modo el Ministerio de Educación Nacional de Colombia (2013)refiere que la inclusión de las TIC han influenciado en nuevas didácticas y han potenciado ideales pedagógicos al ofrecer ambientes de aprendizaje ricos en experiencias, recursos y materiales que cautivan el interés del alumnado, permiten libertad de explorar, analizar y construir conocimiento, así como el poseer múltiples fuentes de información actualizadas constantemente.

Es este el cambio que esperamos promover, para que nuestros docentes se incorporen y utilicen la estrategia de enseñanza asistida por herramientas de información y comunicación. 
Competencia digital y desarrollo profesional de los docentes de dos instituciones de educación básica regular del distrito de Los Olivos, Lima-Perú

\section{Dimensiones de la competencia digital}

Según la propuesta referida por Prendes, Gutiérrez \& Martínez (2018) la competencia digital presenta cinco dimensiones, (ver tabla 1)

Tabla 1

Dimensiones de la competencia digital según Prendes, et al.

\begin{tabular}{|c|c|}
\hline Dimensiones & Descripción \\
\hline Técnica & $\begin{array}{l}\text { Permite la capacitación y desarrollo para el } \\
\text { dominio de las tecnologías, la información y la } \\
\text { comunicación, con el manejo de herramien- } \\
\text { tas de edición digital, realizar trabajos con } \\
\text { eficacia en contenidos digitales, en entornos } \\
\text { virtuales de enseñanza- aprendizaje. Incide } \\
\text { en la comprensión y uso eficaz de los siste- } \\
\text { mas informáticos, configuración, instalación } \\
\text { y actualización de software o dispositivos } \\
\text { informáticos }\end{array}$ \\
\hline $\begin{array}{l}\text { Informacional } \\
\text { y comunica- } \\
\text { tiva }\end{array}$ & $\begin{array}{l}\text { Engloba la comunicación interpersonal y } \\
\text { social, obtención, evaluación, organización y } \\
\text { el tratamiento de la información en entornos } \\
\text { digitales, para lo que se requiere, usar siste- } \\
\text { mas informáticos, usar motores de búsqueda, } \\
\text { guardar, archivar y recuperar información en } \\
\text { formato digital, evaluar la calidad y pertinencia } \\
\text { de la información }\end{array}$ \\
\hline Educativa & $\begin{array}{l}\text { Relacionada a la orientación, guía y } \\
\text { evaluación de los procesos de construcción } \\
\text { del conocimiento, así como la colaboración } \\
\text { en entornos digitales, a través de dispositi- } \\
\text { vos digitales, velando por la calidad de los } \\
\text { contenidos }\end{array}$ \\
\hline Analítica & $\begin{array}{l}\text { Tiene como componente la capacidad de } \\
\text { reflexionar de modo crítico sobre nuestra } \\
\text { propia competencia, las posibilidades de } \\
\text { asumir todos los elementos que constituyen el } \\
\text { universo de las tecnologías de comunicación, } \\
\text { para desarrollar los procesos de interacción, } \\
\text { colaboración y evaluación de los aprendizajes }\end{array}$ \\
\hline Social y ética & $\begin{array}{l}\text { Hace referencia a la elaboración en forma } \\
\text { colectiva, trabajando en equipo, en redes } \\
\text { y espacios tele-colaborativos y sobre todo } \\
\text { contribuir al aprendizaje mutuo, al actuar de } \\
\text { forma responsable, seguro y cívico, a través } \\
\text { del respeto a la privacidad, la seguridad de } \\
\text { los datos personales y la información en } \\
\text { internet, con conocimiento de la norma legal } \\
\text { que ampara los derechos de propiedad, } \\
\text { como inicio del ejercicio responsable de la } \\
\text { ciudadanía digital }\end{array}$ \\
\hline
\end{tabular}

Fuente: Prendes et al (2018, p. 9)

Se considera, a su vez el modelo que dimensiona Boris (2009, p. 14) que considera las cinco dimensiones concordantes, (Ver Tabla 2)
Tabla 2

Dimensiones de la competencia digital según Boris.

\begin{tabular}{ll}
\hline Dimensiones & Descripción \\
\hline Aprendizaje & $\begin{array}{l}\text { Referida a la transformación de la información } \\
\text { en conocimiento y su adquisición }\end{array}$ \\
Informacional & $\begin{array}{l}\text { Precisa la obtención, la evaluación y el trata- } \\
\text { miento de la información en entornos digitales. }\end{array}$ \\
Comunicativa & $\begin{array}{l}\text { Abarca la comunicación interpersonal y la } \\
\text { social. }\end{array}$ \\
Cultura digital & $\begin{array}{l}\text { Hace referencia a las prácticas sociales y } \\
\text { culturales de la sociedad del conocimiento y la } \\
\text { ciudadanía digital. }\end{array}$ \\
Tecnológica & $\begin{array}{l}\text { Referida a la alfabetización tecnológica y el co- } \\
\text { nocimiento y dominio de los entornos digitales. }\end{array}$ \\
\hline
\end{tabular}

Fuente: Boris, (2009)

\section{Desarrollo profesional docente.}

El desarrollo docente es un constante aprendizaje y una permanente actualización y renovación de conocimiento, habilidades e innovación, tanto para el uso de estrategias como el mejoramiento de capacidades educativas, como lo señala Osorio (2016)

Existe el constructo en el imaginario de las personas que quienes ostentan estudios de pre y posgrado pueden resolver toda la formación de los profesionales. Sin embargo, lo cierto es que esta formación está limitada hacia los docentes, ya que con supuestos modelos ideales sean aplicados en sesiones [...] la formación docente debe estar orientada a ayudar a superar el desgaste que sufren los profesores, en cuanto a su aprendizaje en su formación inicial y lo nuevo que deben aprender en el desempeño de sus funciones como docente, y que esto les permita estar a la par con la evolución en la sociedad. (p. 41)

Vaillant (2016) refiere que hay un énfasis en mantener la calidad del profesorado, por lo que hay una preocupación en fortalecer el desarrollo profesional del docente, y que este debe estar establecido a través de políticas públicas que aseguren la continuidad y sostenibilidad de procesos que permitan una formación continua de estos. Para Valdivieso \& Ángeles (2016) la formación profesional docente debe ser específica, en el que implique la apropiación y aplicación de las TIC 
para aprovechar las potencialidades de estas en la práctica docente. Asimismo, que durante todo el tiempo que este continúe con su desarrollo profesional y su formación continua, inicie un proceso que se ajuste a la exigencia de adquirir y mejorar su competencia digital.

En cuanto a entender la complejidad del desarrollo profesional de los docentes Trillo et al. (2017) señalan que este se da bajo la diversidad de tiempos, contextos y experiencias de aprendizaje, así como los contenidos que se tratan y los procesos, todos los cuales son convertidos en aprendizajes por los sujetos en su condición de profesionales, y serán utilizados en su desempeńo, en tiempos y situaciones distintas y cambiantes.

\section{Dimensiones del desarrollo profesional docente.}

A partir de la teoría y la praxis del quehacer educativo, el cual es complejo por naturaleza, es necesario tener un sistema de desarrollo profesional integral y permanente a nivel académico, institucional y personal, ello hace que se formule las siguientes dimensiones:

1. Dimensión Pedagógica, que constituye el núcleo y base fundamental del desenvolvimiento profesional docente. Es un saber específico, construido en la reflexión teórico-práctica, que permite apelar a saberes diversos para cumplir con el rol que tiene este. En los que están incluidos competencias capacidades, actitudes y el compromiso de los estudiantes para aprender y formarse. Ballester \& Sánchez (2011, p. 23) refieren que "La dimensión pedagógica [...] es el conjunto de decisiones articuladas en torno a un modelo educativo basado en competencias básicas y brindan los lineamientos de contexto y contenido en el ámbito pedagógico, en aspectos relacionados a lo organizativo, curricular y didáctico".

Avín et al., (2016, p. 3) hacen mención sobre los aspectos pedagógicos, que son los propósitos y los contenidos de la enseñanza, el entendimiento e identificación del alumno y docente, a la relación pedagógica que se da entre ellos; aunado con el conocimiento, las estrategias didácticas y la evaluación de los aprendi- zajes en todo nivel.

2. Dimensión teleológica, visto a nivel institucional todo docente necesita tener claro su orientación y fin educativo como lo señalan García, Verde et al, (2011, p 57) "La meta social de la docencia consiste en la transmisión de la cultura y la formación de personas críticas", en ese sentido la labor docente tiene un fin inherente a su naturaleza y función, tiene un rol determinado en la línea del cumplimiento de las metas, fines y propósitos educacionales establecidos por el Estado, a través de sus normas y directivas. Montalvo (2012) refiere que esta dimensión, es la lección del profesor, con una visión madura y puesta al día del conocimiento, seleccionando los contenidos posibles y necesarios. Los docentes asumen como reto desarrollar sus competencias como parte de su formación profesional y a los compromisos asumidos con la sociedad, el Estado y el carácter trascedente de ser educador.

3. Dimensión deontológica, que se formula bajo la concepción del desarrollo profesional a partir del respeto y cumplimiento del conjunto de regulaciones, principios y normas que orientan el ejercicio profesional docente, para establecer su línea y orientación. Gonzalo \& Ruiz (2013) mencionan que los principios deontológicos que marcan toda actuación docente, son: Responsabilidad, veracidad, justicia, respeto, empatía, solidaridad, espíritu crítico, desinterés, lo mismos que sirven para que el docente conozca y asuma plenamente sus obligaciones, que la sociedad exige.

Estas dimensiones presentadas por los distintos autores nos ayudaron a sustentar las distintas estrategias de recolección de información y orientar la discusión y conclusiones, para así engranar un plan de trabajo efectivo centrado en el docente como pilar esencial de la educación en nuestro país.

\section{Materiales y Métodos}

\section{Participantes}

La población estuvo constituida por 215 docentes 
Competencia digital y desarrollo profesional de los docentes de dos instituciones de educación básica regular del distrito de Los Olivos, Lima-Perú

de las instituciones educativas públicas "Precursores de la Independencia Nacional" y "Nuestra Señora de Lourdes" distrito de Los Olivos, LimaPerú, considerando como criterios de inclusión, a docentes de educación básica regular, de los niveles primario y secundario, de ambos sexos, de los turnos mañana y tarde. Y como criterios de exclusión a los docentes del nivel inicial, que se encontraban en capacitación y de licencia.

Se aplicó la fórmula de Arkin y Kolton para realizar el análisis de la muestra, dando como resultando 100 docentes. Para la selección de los docentes de la muestra se aplicó la técnica aleatoria simple, ya que todos los participantes del total de ambas instituciones educativas tuvieron la misma probabilidad de ser seleccionados, (Vara, 2015; Hernández, Fernández \& Baptista, (2014). En este estudio no se ha considerado los aspectos sociodemográficos de los docentes, debido a que nuestro interés se centraba en conocer la relación entre competencias digitales y desarrollo profesional docente.

\section{Instrumento}

Para la recolección de los datos se utilizaron dos cuestionarios en escala Likert, para la variable competencias digitales el cuestionario consto de 25 ítems, y tenía un total de tres escalas, desde un buen nivel a un nivel bajo, (Anexo 1) Para la variable Desarrollo profesional docente el cuestionario fue de 20 ítems, baremado con niveles desde alto a bajo. (Anexo 2). En la tabla 3 y 4 se realiza la descripción de ambos cuestionarios.

Tabla 3

Descripción del cuestionario de Competencias digitales

\begin{tabular}{ll}
\hline \multicolumn{2}{c}{ Variable: Competencias digitales } \\
\hline Dimensiones & \multicolumn{1}{c}{ Indicadores } \\
\hline Educativa & $\begin{array}{l}\text { Representa y crea conocimiento en diferentes } \\
\text { lenguajes. }\end{array}$ \\
& $\begin{array}{l}\text { Produce conocimientos y publica información } \\
\text { Lleva a cabo proyectos, resuelve problemas y } \\
\text { toma decisiones en entornos digitales. }\end{array}$ \\
& Trabaja con eficacia con contenidos digitales \\
& Hace uso de las TIC como instrumento del \\
& pensamiento reflexivo y crítico.
\end{tabular}

\begin{tabular}{|c|c|}
\hline & Variable: Competencias digitales \\
\hline Dimensiones & Indicadores \\
\hline Analítica & $\begin{array}{l}\text { Usa sistemas informáticos y navega por Internet } \\
\text { Utiliza diferentes fuentes y motores de búsque- } \\
\text { da } \\
\text { Guarda, archiva y recupera la información } \\
\text { Conoce y utiliza herramientas y recursos } \\
\text { Evalúa la calidad, la pertinencia y la utilidad de } \\
\text { la información. }\end{array}$ \\
\hline $\begin{array}{l}\text { Informacio- } \\
\text { nal y comuni- } \\
\text { cativa }\end{array}$ & $\begin{array}{l}\text { Se comunica mediante dispositivos digitales y } \\
\text { software específico. } \\
\text { Verifica la calidad y el contenido de la comuni- } \\
\text { cación } \\
\text { Emplea herramientas de elaboración colectiva } \\
\text { Participa proactivamente en entornos virtuales. } \\
\text { Colabora y contribuye al aprendizaje mutuo con } \\
\text { herramientas digitales. }\end{array}$ \\
\hline Social y ética & $\begin{array}{l}\text { Gestiona la identidad digital y el grado de priva- } \\
\text { cidad y de seguridad de los datos personales. } \\
\text { Respeta los diferentes ámbitos de propiedad de } \\
\text { los contenidos digitales. } \\
\text { Reflexiona sobre la dimensión social y cultural } \\
\text { de la sociedad del conocimiento. } \\
\text { Propicia el ejercicio responsable de la ciudada- } \\
\text { nía digital. }\end{array}$ \\
\hline Técnica & $\begin{array}{l}\text { Comprende y utiliza con eficacia los dispositivos } \\
\text { Utiliza las funciones de navegación } \\
\text { Determina y configura el software y el entorno } \\
\text { de trabajo. } \\
\text { Instala, actualiza y desinstala software }\end{array}$ \\
\hline
\end{tabular}

Fuente: Prendes et al., (2018)

Tabla 4

Descripción de Desarrollo profesional docente

\begin{tabular}{ll}
\hline \multicolumn{2}{c}{ Variable: Desarrollo profesional docente } \\
\hline Dimensiones & \multicolumn{1}{c}{ Indicadores } \\
\hline Pedagógica & $\begin{array}{l}\text { Demuestra el saber pedagógico construido } \\
\text { en la reflexión teórico-práctica, que le permite } \\
\text { apelar a saberes diversos para cumplir su rol. }\end{array}$ \\
Teleológica & $\begin{array}{l}\text { Reflexiona sobre el objetivo de la educación } \\
\text { basados en el respeto a los principios demo- } \\
\text { cráticos de convivencia y a los derechos y } \\
\text { libertades fundamentales. }\end{array}$ \\
Deontológica & $\begin{array}{l}\text { Conoce y practica los principios y normas } \\
\text { éticas de la profesión docente. }\end{array}$ \\
\hline
\end{tabular}

Fuente: Elaboración propia (2018)

Para ambos instrumentos se procedió a establecer la validez de contenido, mediante el juicio de 7 expertos, quienes concuerdan que los instrumentos presentan un grado mayor al 0.75 , utilizando el método Delphi. (Ver tabla 5 y 6 ) 


\section{Tabla 5}

Validez de contenido por criterio de jueces: Competencias digítales. valor máximo 1 y mínimo 0

\begin{tabular}{|c|c|c|c|c|c|c|c|c|}
\hline \multirow{3}{*}{$\begin{array}{l}\text { Dimen- } \\
\text { siones }\end{array}$} & \multicolumn{7}{|c|}{ Calificación de los jueces } & Índice \\
\hline & $\mathrm{J}$ & $J$ & $\mathrm{~J}$ & $\mathrm{~J}$ & $J$ & . & J & \\
\hline & 1 & 2 & 3 & 4 & 5 & 6 & 7 & \\
\hline 1 & 0 & 1 & 1 & 1 & 1 & 1 & 1 & 0.86 \\
\hline 2 & 1 & 1 & 1 & 1 & 1 & ( & 1 & 0.86 \\
\hline 3 & 1 & 1 & 1 & 1 & 1 & 1 & 1 & 1.00 \\
\hline 4 & 1 & 1 & 1 & 1 & 1 & 1 & 0 & 0.8 \\
\hline
\end{tabular}

Fuente: Elaboración propia (2018)

\section{Tabla 6}

Validez de contenido por criterio de jueces: Desarrollo profesional docente

\begin{tabular}{|c|c|c|c|c|c|c|c|c|c|}
\hline \multirow{3}{*}{$\begin{array}{l}\text { Dimen- } \\
\text { siones }\end{array}$} & \multicolumn{7}{|c|}{ Calificación de los jueces } & \multirow{3}{*}{$\begin{array}{l}\text { Índice } \\
\text { de eva- } \\
\text { luación }\end{array}$} & \multirow[t]{3}{*}{ Sugerencias } \\
\hline & $\mathrm{J}$ & $\mathrm{J}$ & $\mathrm{J}$ & $\mathrm{J}$ & $\mathrm{J}$ & J & J & & \\
\hline & 1 & 2 & 3 & 4 & 5 & 6 & 7 & & \\
\hline 1 & 1 & 1 & 1 & 1 & 1 & 1 & 1 & 1.00 & \\
\hline 2 & 1 & 1 & 1 & 1 & 1 & 0 & 1 & 0.86 & $\begin{array}{l}\text { Cambiar en el ítem: Parti- } \\
\text { cipa en las capacitaciones } \\
\text { referidas al manejo de las } \\
\text { nuevas tecnologías por } \\
\text { "Participa en las capacita- } \\
\text { ciones referidas al manejo } \\
\text { de las nuevas tecnologías, } \\
\text { para su desempeño do- } \\
\text { cente" para completar la } \\
\text { información que se busca } \\
\text { recoger del ítem. }\end{array}$ \\
\hline
\end{tabular}

Para la confiabilidad, se empleó el coeficiente Alfa de Cronbach, cuyos resultados fueron 0.77 para Competencias digitales y 0.75 para Desarrollo profesional docente, lo que significa que los dos instrumentos alcanzan una excelente confiabili- dad, previo de la validez de contenido; donde el informante técnico asumió 1 en señal de su conformidad mientras 0 en señal de desconformidad, lo cual se aprecia en la Tabla 7.

Tabla 7

Confiabilidad de los instrumentos

\begin{tabular}{llcc}
\hline \multicolumn{1}{c}{ Cuestionarios } & $\begin{array}{c}\text { Validez de } \\
\text { contenido }\end{array}$ & $\begin{array}{c}\text { Alfa de } \\
\text { Cronbach } \\
\text { (confiabilidad) }\end{array}$ & $\begin{array}{c}\mathrm{N}^{\circ} \text { de } \\
\text { items }\end{array}$ \\
\hline $\begin{array}{l}\text { Cuestionario sobre la } \\
\text { Competencia digital }\end{array}$ & Aplicable & 0.77 & 25 \\
\hline $\begin{array}{l}\text { Cuestionario sobre el } \\
\text { Desarrollo profesional } \\
\text { docente }\end{array}$ & Aplicable & 0.75 & 20 \\
\hline
\end{tabular}

Fuente: Elaboración propia (2018)

Tipo y diseño

El tipo de estudio es básico, de acuerdo a Vara (2015) permite generar nuevas formas de interpretar el objeto de estudio y facilita el acercamiento a la realidad de estudio y se asumen teorías que explican las variables analizadas.

El diseño es no experimental, de corte transversal, correlacional causal, de acuerdo a Hernández, et al. (2014), los diseños no experimentales se caracterizan por observar los fenómenos sin intervención del investigador, es transversal al recopilar información en un determinado momento y correlacional causal, al describir una relación de causalidad entre las variables de estudio.

Se empleó el método hipotético deductivo, consiste en partir de afirmaciones o supuestos para refutar o falsear tales hipótesis, infiriendo de ellas conclusiones que se confrontan con los hechos; es decir, comprobar la veracidad o falsedad de los supuestos planteados, que no se pueden demostrar directamente, debido a su carácter de enunciado general. (Bernal et al., 2014).

\section{Procedimiento}

El estudio fue realizado a través de etapas secuenciales:

- Etapa de inicio: La investigación inicio con la formulación del problema a nivel institucio- 
Competencia digital y desarrollo profesional de los docentes de dos instituciones de educación básica regular del distrito de Los Olivos, Lima-Perú

nal, de la cual se tuvo la idea de investigación, lo que finalmente se constituyó en el tema y posterior título de investigación.

- Etapa segunda: La existencia del problema se consultó con la revisión de la literatura especializada, en bases de datos, revistas electrónicas y libros.

- Etapa tercera: Se planteó interrogantes de investigación, objetivos e hipótesis producto de la problemática encontrada.

- Etapa Cuarta: Se desarrolló todo el soporte teórico, lo que permitió la operacionalización de las variables, para la elaboración de los cuestionarios: competencias digitales y desarrollo profesional, pasando los mismos por los procesos de validez de contenido y un proceso estadístico, que se realizó en un grupo piloto, para poder realizar el análisis estadístico y determinar la confiabilidad de los instrumentos. Considerando que cumplía con las condiciones se procedió a recoger los datos de la muestra.

- Etapa quinta: La muestra fue convocada para explicarles el objetivo del estudio y para la firma del consentimiento informado. Posteriormente para la aplicación de los instrumentos, en la que se dio lectura a las instrucciones.

- Etapa sexta: Se procesó los datos recogidos de la muestra de estudio, mediante el programa SPSS, V_24. Para la presentación de los niveles de ambas variables, se utilizó niveles porcentuales. Para determinar la asociación entre variables se utilizó el estadístico Chi cuadrado. Respondiendo al diseño del estudio, correlacional causal, en su primera presentación, la correlación se realizó con los estadísticos ordinales, rho de Spearman, Kendall y Gamma. La relación causal se obtuvo con el coeficiente de determinación Nagelkerke, mostrándose los porcentajes de incidencia

- Etapa de cierre: Finalmente se desarrolló la discusión y conclusiones de la investigación.

\section{Confidencialidad o consentimiento informado}

Se realizó el estudio solicitando la autorización a través de cartas de permiso correspondiente a las instituciones educativas, donde se exponía el porqué de la investigación. Una vez obtenida la aceptación, se convocó a la muestra previamente seleccionada siguiendo rigurosamente los criterios de inclusión, para solicitar el consentimiento informado, a través del cual, eran conscientes que ser parte de un estudio que aporta a la solución del problema y contribuye al conocimiento científico, Anexo 3.

\section{Resultados}

Se obtuvieron y procesaron dos bloques de resultados; en la primera parte se presenta los resultados descriptivos, con los niveles de las variables (nivel alto, regular, etc.), establecidos en el proceso de operacionalización y la aplicación del baremo, según las respuestas de la muestra de estudio en una tabla de contingencia de las frecuencias y porcentajes, (Tabla 8). Y el segundo bloque se presentan los coeficientes de correlación, (Tabla 9).

\section{Tabla 8}

Niveles de la Competencia digital y el Desarrollo profesional docente de EBR de la Red 02 UGEL 02.

\begin{tabular}{|c|c|c|c|c|c|}
\hline & & & \multicolumn{2}{|c|}{$\begin{array}{l}\text { Competencia } \\
\text { digital }\end{array}$} & \multirow[b]{2}{*}{ Total } \\
\hline & & & Regular & Bueno & \\
\hline \multirow{5}{*}{$\begin{array}{l}\text { Desarrollo } \\
\text { profesional }\end{array}$} & \multirow[t]{2}{*}{ Moderado } & $\mathrm{Fi}$ & 51 & 3 & 54 \\
\hline & & pi $\%$ & $51 \%$ & $3 \%$ & $54 \%$ \\
\hline & \multirow[t]{3}{*}{ Alto } & $\mathrm{Fi}$ & 27 & 19 & 46 \\
\hline & & & $27 \%$ & $19 \%$ & $46 \%$ \\
\hline & & $\mathrm{Fi}$ & 78 & 22 & 100 \\
\hline Total & & $\mathrm{Fi} \%$ & $78 \%$ & $22 \%$ & $100 \%$ \\
\hline
\end{tabular}

Chi cuadrado X2 = 18.499; gl=1; sig. asin. bilateral $=0.00$ Fuente: Elaboración propia (2018)

En la Tabla 8 se muestran los resultados por niveles de las competencias digitales y el desarrollo profesional de los docentes; observándose una tendencia de complementariedad entre ellos. Con respecto a las competencias digitales, el $78 \%$ de los docentes presentan un nivel regular, y el 22\% un buen nivel, con respecto al desarrollo profesional; el 54\% de los docentes presentan un nivel moderado y el $46 \%$ alto nivel. Sin embargo, el $51 \%$ de los docentes presentan regular nivel en competencias digitales puesto que muestran moderado nivel en su desarrollo profesional, y 
el 19\% de los docentes muestran buen nivel en competencias digitales y presentan alto nivel de desarrollo profesional, expresado en la Figura 1. Estos resultados permiten afirmar que existe correlación entre las competencias digitales y el desarrollo profesional docente determinado por el Chi cuadrado X2c=18.499 con un 1 grado de libertad y el valor de significación estadística de p_valor 0.00 a un nivel de confianza del $95 \%$.

Niveles de competencia digital y desarrollo profesional docente

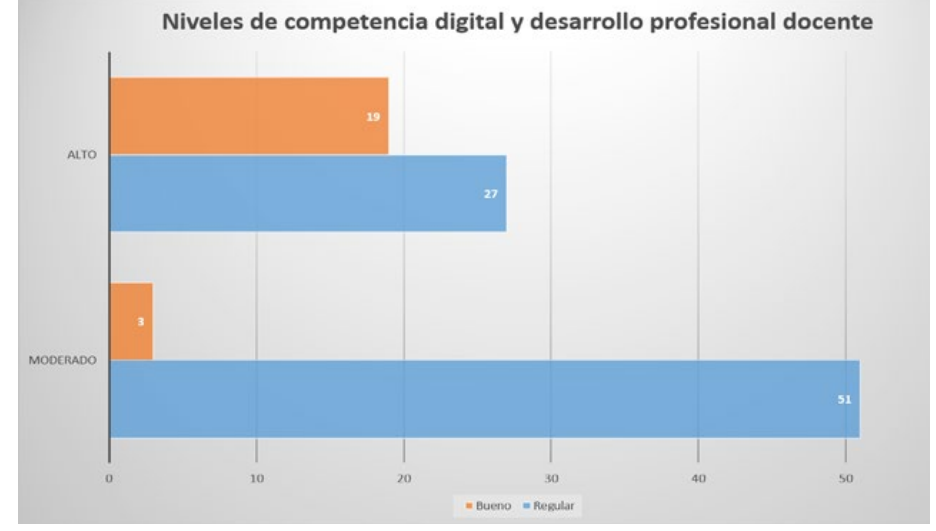

Figura 1

Registros de Educación Básica Regular del distrito de Los Olivos, Lima-Perú.

Las variables de estudio, por su naturaleza son de tipo cualitativa, de escala ordinal; y los coeficientes de correlación reafirman la existencia de concordancia y complementariedad entre ellos. A continuación, se muestra la relación entre las variables de estudio:

Tabla 9

Coeficiente de correlación entre las competencias digitales y el desarrollo profesional.

\begin{tabular}{llcc}
\hline \multicolumn{3}{c}{ Medidas simétricas-Correlaciones } \\
\hline & $\begin{array}{l}\text { Pruebas de } \\
\text { asociación }\end{array}$ & Valor & $\begin{array}{l}\text { Significación } \\
\text { aproximada }\end{array}$ \\
\hline $\begin{array}{l}\text { Ordinal por } \\
\text { ordinal }\end{array}$ & $\begin{array}{l}\text { Rho de } \\
\text { Spearman }\end{array}$ & $.416^{* *}$ & .000 \\
\cline { 2 - 4 } & $\begin{array}{l}\text { Tau-b de } \\
\text { Kendall }\end{array}$ & $.430^{* *}$ & .000 \\
\cline { 2 - 4 } & Gamma & $.846^{* *}$ & .000 \\
\hline $\begin{array}{l}\text { N. }{ }^{\circ} \text { de casos } \\
\text { válidos }\end{array}$ & 100 & \\
\hline
\end{tabular}

Fuente: Elaboración propia (2018)

Los resultados de la tabla 9 permiten reafirmar que existe una relación directa y moderada como se observa mediante el coeficiente de correlación para escala ordinal, Rho de Spearman 0.416 , asimismo, el valor de Tau-b de Kendall 0.430; pensando que existieran o no rangos empatados se utilizó el coeficiente de Gamma, con un máximo valor de 0.846; este último estadístico permitió afirmar la correlación entre variables categóricas, (Véliz, 2014). A medida que se incrementa el nivel de las competencias digitales para su labor docente, se manifiesta alto nivel de desarrollo profesional.

Resultados de parámetros y contrastación de hipótesis.

Se tomó de manera porcentual, en referencia al mayor nivel de la variable dependiente, la cual permite determinar el pronóstico porcentual. Sin embargo, para el proceso inferencial con respecto a la contrastación de la hipótesis del estudio, se procedió a analizar con regresión logística ordinal; lo que permitió mostrar la predicción porcentual a partir del Pseudo $\mathrm{R}$ cuadrado asociado al estadístico de Wald a un nivel de confianza del 0.05; al respecto la hipótesis del estudio demostró que existe relación de la competencia digital y el desarrollo profesional de los docentes de Educación Básica Regular de la Red 08 de la UGEL 02, de Lima $s$ lo cual fue contrastado con las pruebas estadísticas que a continuación se describen en las Tablas 10 y 11 .

En la Tabla 10 se muestran los coeficientes de la regresión logística ordinal, en el primer bloque, la estimación de la competencia digital frente al desarrollo profesional; al respecto se tiene, el coeficiente de estimación protector de -2.482 , $(\exp -2.482=$ 0.0836 ), implica estadísticamente, que los docentes con nivel regular en el uso de competencia digital, tienen la posibilidad del $8.357 \%$ de mostrar alto nivel en su desarrollo profesional docente, quien es reafirmado por el coeficiente de restricción de Wald, el valor de significación estadística p_valor de 0.000 . Sin embargo, el $24 \%$ del desarrollo profesional se debe o es explicada por las competencias digitales de los docentes, determinado por el coeficiente del seudo R- cuadrado de Nagalkerke. 
Competencia digital y desarrollo profesional de los docentes de dos instituciones de educación básica regular del distrito de Los Olivos, Lima-Perú

\section{Tabla 10}

Estimación de los parámetros logísticos de las competencias digitales y el desarrollo profesional

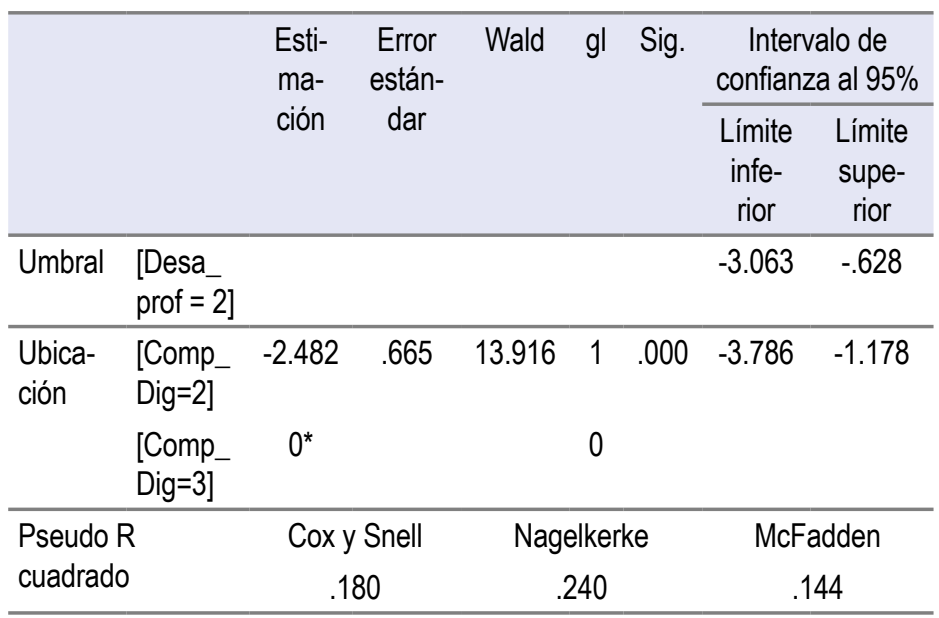

Fuente: Elaboración propia (2018)

* Este parámetro está establecido en cero porque es redundante.

\section{Tabla 11}

Estimación de los parámetros logísticos de las competencias digitales en las dimensiones del desarrollo profesional docente. presentan los docentes, se tiene la probabilidad del buen desarrollo profesional docente, (Hosmer \& Lemeshow, 2000). La significancia individual de cada variable independiente, generalmente, se analiza a través de la prueba de Wald que sigue el comportamiento del Chi cuadrado X2 $(1 ; 0.05)$ y los coeficientes del pseudo $\mathrm{R}$ cuadrado de $\mathrm{Na}$ galkerke para las dimensiones: pedagógica, teleológica y deontológica; esta última presenta mayor incidencia, por cuanto el $52.9 \%$ del comportamiento de la dimensión deontológica es explicada por las competencias digitales durante su desarrollo profesional del docente y el $47.1 \%$ se debe a otros factores. Podemos notar que existe certeza para deducir que existe evidencia estadísticamente significativa para afirmar que los docentes con competencias digitales alcanzan un desarrollo profesional por encima de la media.

\section{Discusión y Conclusiones}

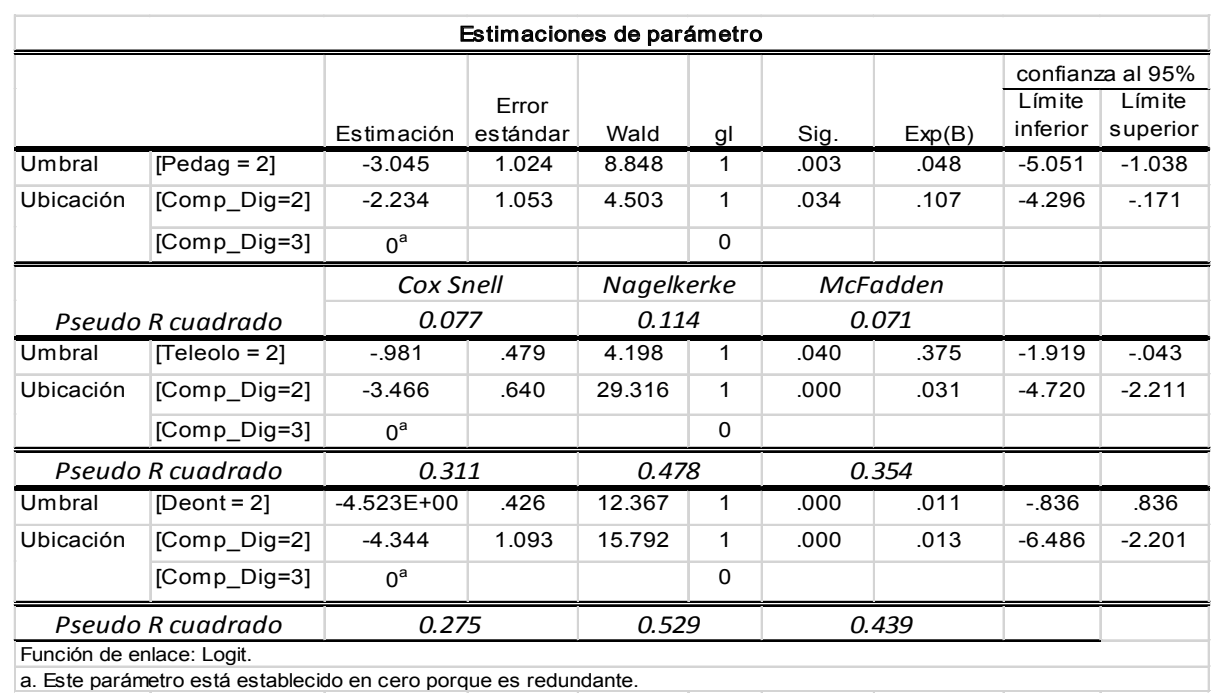

A partir de la obtención de los datos y su procesamiento, se obtuvo el coeficiente del seudo Rcuadrado, de Nagalkerke, donde el $24 \%$ del desarrollo profesional es explicado por las competencias digitales de los docentes y el $76 \%$ se debe a otros factores. Así también en cuanto a los resultados descriptivos, con respecto a las competencias digitales, el $78 \%$ de los docentes presentan un nivel regular Por tanto es estadísticamente sustentable señalar que la mayoría de los docentes Fuente: Elaboración propia (2018)

Los resultados de la Tabla 11 indican que el valor de significación estadística (sig. p_valor) sea menor a 0.05 , es que la ecuación de la regresión logística ordinal alcanza buen ajuste, (Agresti, 1990). Los coeficientes negativos de los estimadores hacen que el Odds Ratio (ExpB) sea menor a uno, y al no estar definida la unidad dentro del intervalo de confianza al 95\%; nos permite estadísticamente afirmar que al mejorar los niveles regulares de las competencias digitales que disponen de un nivel alto de competencia digital concordantes a lo que refiere, Esteve (2015) y el $22 \%$ un buen nivel de competencias digitales, ello permite corroborar la afirmación referida por García (2017) que se promueva el uso de las TIC, en la búsqueda y procesamiento de información, lo que nos indica que los docentes poseen cierto conocimiento en el uso y apropiación de las tecnologías, y por ende haber adquirido un mínimo de competencia digital.

Con respecto al desarrollo profesional, se obtiene 
que un $46 \%$ de los docentes percibe que existe un alto nivel, existe la necesidad de resaltar el uso de las estrategias implementadas en este campo del quehacer educativo teniendo como principal objeto de estudio al docente, por ello se hace necesario apostar por una formación del profesorado que asuma la relevancia de la competencia digital y que tome como punto de partida una adecuada comprensión de lo que supone ser un docente competente digitalmente. Aspecto que Enríquez (2014) precisa en su estudio que el docente está en un continuo desarrollo profesional, al ser este un proceso de crecimiento permanente y dinámico. En tal sentido los resultados de la investigación corroboran nuestro objetivo general, así como los resultados de los trabajos previos. Los datos permiten afirmar que existe correlación entre las competencias digitales docente y el desarrollo profesional, determinado por el Chi cuadrado $X 2 c=18.499$ con un 1 grado de libertad y el valor de significación estadística de p_valor 0.00 a un nivel de confianza del $95 \%$, por ende, el mejoramiento de la educación básica y la calidad de esta depende entre otros factores del manejo adecuado de las TIC y las competencias digitales de los profesores, (Vaillant, 2016).

Finalmente, a partir de los hallazgos se recomienda aunar esfuerzos y desde la academia preparar a los docentes en el dominio de herramientas básicas de tecnologías de información y comunicación y así desarrollar sus competencias digitales, logrando asi un impacto mayor en su exitoso desarrollo profesional, lo cual es un desafío constante y un asunto clave, con mayores exigencias y posibilidades de alcanzar niveles de excelencia en este siglo XXI. Así como, incorporar en el plan de estudios de los futuros profesores nuevos perfiles vinculados a la integración, dominio de las TIC y a su utilización en el ámbito educativo, que nos exigen los tiempos actuales; en referencia a lo que precisa el Ministerio de Educación, (2013), a través de talleres y/o formación continua, la misma que redundará en el desarrollo profesional y la mejora de praxis profesional en el aula.

Para futuros estudios se propone incluir aspectos sociodemográficos que verifiquen si estos tienen alguna incidencia en el desarrollo de sus competencias digitales y mejora su desarrollo profesional.

\section{REFERENCIAS BIBLIOGRÁFICAS}

Agresti, A. (1990). Categorical Data Analysis. New York: John Wiley \& Sons.

Almerich, G., Díaz-García, I., Cebrián-Cifuentes, S. \& Suaréz-Rodriguez, J. (2018) Estructura dimensional de las competencias del siglo XXI en alumnado universitario de educación. RELIEVE, 24(1), 1-21. https://doi.org/10.7203/ relieve.24.1.12548

Avín R.; Llanos L, Morales I., Santamarìa B. \& Soto Z. (2016) Dimensión pedagógica de las instituciones educativas. Universidad Pedagógica Nacional, México.

Ballester, M. \& Sánchez, J. (2011) La dimensión pedagógica del enfoque de competencias en educación obligatoria. Ensayos, 26, 17-34.

Bernal, C., Correa, A., Pineda, M.; Lemus, Fonseca, F. \& Muñoz, C. (2014). Fundamentos de la investigación. Primera edición. México: Pearson Educación.

https://doi.org/10.29097/2011-639X.169

Boris, M. (2009) La competencia digital, una propuesta. http://www.xtec.es/-bmir/

Caudillo, Y. (2016). Competencia Digital en el Proceso de Apropiación de las TIC en Jóvenes de Secundaria en el Estado de Sonora, México. Propuesta de Innovación Educativa para la Mejora de las Habilidades Digitales en el Aula. México. Recuperado de http://www.pics.uson.mx/doctorado/ wp-content/uploads/2018/02/Caudillo-Ruiz-Tesis-doctorado.pdf

Chapilliquen, M. (2016) Competencias digitales en estudiantes con diferentes estilos de aprendizaje, del séptimo ciclo de educación secundaria, desarrolladas a través de la red social educativa Edmodo en una institución educativa pública de la Unidad de Gestión Educativa Local $N^{\circ} 03$, el año 2015. Pontificia Universidad Católica del Perú, Lima-Perú.

CNE-Consejo Nacional de Educación (2006) Proyecto Educativo Nacional al 2021. La educación que queremos para el Perú. Recuperado de http://repositorio.minedu.gob.pe/ bitstream/handle/123456789/304/166.\%20Proyecto\%20 Educativo\%20Nacional\%20al\%202021.\%20La\%20educaci\%C3\%B3n\%20que\%20queremos\%20para\%20el\%20 Per\%C3\%BA.pdf?sequence $=1 \&$ isAllowed $=y$

Colás, M., De Pablos, J. \& Ballesta, J. (2018) Incidencia de las TIC en la enseñanza en el sistema educativo español: una revisión de la investigación. https://doi.org/10.6018/ $\mathrm{red} / 56 / 2$

Colomer, J.; Hernández H. \& Ketil B. (2018) Competencia digital docente Perspectivas y prospectivas para una nueva escuela. Revista Comunicar. España Recuperado de http:// tesis.pucp.edu.pe/repositorio/handle/123456789/6744

Durán, M. C., Prendes, M.P.E. \& Gutiérrez, I. P. (2019). Certificación de la Competencia Digital Docente: propuesta para el profesorado universitario. RIED. Revista Iberoamericana de Educación a Distancia, 22(1), 187-205. https://doi. org/10.5944/ried.22.1.22069 
Competencia digital y desarrollo profesional de los docentes de dos instituciones de educación básica regular del distrito de Los Olivos, Lima-Perú

Elacqua, G., Hincapié, D., Vegas, E., \& Alonso, M. (2018) Profesión: profesor en América Latina. ¿Por qué se perdió el prestigio docente y cómo recuperarlo? Washington, D.C. BID. https://doi.org/10.18235/0001172

Enríquez, L. (2014) Concepciones y factores influyentes en el desarrollo profesional docente en España, Chile y Colombia en los últimos diez años Tesis magistral. Pontificia Universidad Católica del Perú. Recuperado de http://tesis.pucp. edu.pe/repositorio/handle/123456789/6744

Esteve, F. (2015) La competencia digital docente. Italia. Universitat Rovira i Virgili.

Esteve, F. (2016) Bolonia y las TIC: de la docencia $1.0 \mathrm{al}$ aprendizaje 2.0. La cuestión universitaria. Universidad Politécnica de Madrid. Recuperado de http://polired.upm.es/ index.php/lacuestionuniversitaria/article/view/3337

García, F. (2017) Competencias digitales en la docencia universitaria del siglo XXI (Tesis doctoral). España. Universidad Complutense de Madrid

García, R., Verde, I \& Vásquez V. (2011). ¿Por qué es necesario trabajar la dimensión ética en la docencia? Universidad de Barcelona. España.

Gonzalo, J. \& Ruiz, M. (2013) El código deontológico de la profesión docente: evolución y posibilidades. Recuperado de: file://C:/Users/maestria-54/Downloads/Dialnet-ElCodigoDeontologicoDeLaProfesionDocente-4406388.pdf

Hernández, R., Fernández, C. \& Baptista, P. (2014). Metodología de la Investigación. México: Mc Graw Hill Education

Hosmer, D. \& Lemeshow, S. (2000). Applied Logistic Regression. New York: John Wiley \& Sons. https://doi. org/10.1002/0471722146

León, P. (2011). Efectos de la estrategia didáctica funcional basado en las TICS sobre el conocimiento procedimental del trabajo de investigación de los estudiantes de post grado de la Universidad César Vallejo filial Pasco V y Ayacucho VIII. 2009. Tesis de Doctorado en Educación. Universidad César Vallejo, Lima Perú

Lion, C. (2012). Desarrollo de competencias para portales de la región. Buenos Aires: RELPE.

Lizcano, R. et al. (2017) Competencias Digitales, Innovación y Prospectiva Colombia. Editorial CIMTED.

Marc, M. (2015). La competencia digital docente. Análisis de la autopercepción y evaluación del desempeño de los estudiantes universitarios de educación por medio de un entorno 3D, Tesis doctoral. Univertitat Rovira I Virgili

Marcelo, C. (2009). La evaluación del desarrollo profesional docente: de la cantidad a la calidad. Universidad de Sevilla. España

Marqués, P. (2014) Competencias docentes en la Era Internet (v. 6.0) Uruguay DIM-EDU.

Mineduc-Ministerio de educación de Chile. (2016) 10 claves sobre el desarrollo profesional docente. Centro UC. Chile
Minedu-Ministerio de Educación (2013). Marco del buen desempeño docente. Lima Perú.

Minedu-Ministerio de educación del Perú, (2016) El impulso de una carrera. Política de revalorización docente en el Perú. Ministerio de Educación del Perú.

Ministerio de Educación Cultura y Deporte-MECD (2017). Marco común de competencia digital docente V 2.0. INTEF. Gobierno de España.

Ministerio de Educación Nacional (2013) Competencias TIC para el desarrollo profesional docente. Oficina de Innovación Educativa con Uso de Nuevas Tecnologías, Ministerio de Educación Nacional, gobierno de Colombia.

Ministerio de Educación y Cultura - MEC (2013) Desarrollo Profesional Docente y Mejora de la Educación. Informe País Universidad de la República Uruguay. MEC. ANEP. UdelaR

Montalvo, A. (2012) Visión teleológica de la enseñanza. Recuperado de: file://C:/Users/maestria-54/Downloads/Dialnet-VisionTeleologicaDeLaEnsenanza-4679768\%20(1).pdf

Osorio, A. (2016). El desarrollo profesional docente en educación básica primaria. Revista latinoamericana de Estudios Educativos, 12(1).

Prendes, M., Gutiérrez, I. \& Martínez, F. (2018). Competencia digital: una necesidad del profesorado universitario en el siglo XXI. RED. https://doi.org/10.6018/red/56/7

Sámano, G.; Reyes, G. \& Robles, H. (2018) Programa de alfabetización digital para formadores de docentes. Recuperado de http://www.rediech.org/ojs/2017/index.php/recie/ article/view/340/432

Trillo, F; Nieto, J.; Martínez, B. \& Escudero. J. (2017) El Desarrollo Profesional y la Colaboración Docente: Un Análisis Situado en el Contexto Español de las Tensiones y Fracturas Entre la Teoría y la Práctica. https://doi.org/10.14507/ epaa. 25.3316

UNESCO. (2010) Towards Inclusive Knowledge Societies. Recuperado de http://uis.unesco.org/sites/default/files/ documents/towards-inclusive-knowledge-societies-a-review-of-unescos-action-in-implementing-the-wsis-outcomes-inclusive-knowledge-societies-wsis-communicationict-2010-en.pdf

Vaillant, D. (2016) El fortalecimiento del desarrollo profesional docente: una mirada desde Latinoamérica. Journal of supranational policies of education.

Valdivieso T., \& Ángeles M. (2016) Competencia digital docente: ¿¿Dónde estamos? Perfil del docente de educación primaria y secundaria. El caso de Ecuador.

Valdivieso-Guerrero, T. (2013) Modelo de competencias digitales y estándares de formación aplicables a docentes del nivel de educación general básica de Latinoamérica. EDUTEC.

Valencia, T. \& Serna, A. (2016). Competencias y estándares TIC. Dimensión pedagógica. Pontificia Universidad Javeriana - Cali. Colombia

Vara, A (2015). Siete pasos para elaborar una tesis. Perú: 
Editorial Macro.

Vargas-D Uniam, Chumpitaz-Campos, Suárez-Díaz \& Badia (2014) Relación entre las competencias digitales de docentes de educación básica y el uso educativo de las tecnologías en las aulas. Profesorado, 18 (3), 361-376.

Veas, J. (2016) Sistema de desarrollo profesional docente. CPEIP. Chile

Véliz, C. (2014). Estadística para la administración y los negocios. Edit. Pearson, Pontificia Universidad Católica del Perú - CENTRUM. Lima -Perú.

Zavala. D.; Muñoz. K, \& Lozano. E, (2016) Un enfoque de las competencias digitales de los docentes. Revista Publicando, 3(9). 
Competencia digital y desarrollo profesional de los docentes de dos instituciones de educación básica regular del distrito de Los Olivos, Lima-Perú

\section{Anexo I \\ CUESTIONARIO SOBRE COMPETENCIA DIGITAL}

\section{Instrucciones:}

Estimado docente, la presente encuesta tiene el propósito de recoger información sobre la competencia digital. Mucho le agradeceré marcar con un aspa "X" en el recuadro que corresponda según su percepción. Esta encuesta tiene el carácter de anónimo, y su procesamiento será reservado, por lo que le solicitamos sinceridad en las respuestas.

\begin{tabular}{cccc}
\hline 1 & 2 & 3 & 4 \\
\hline Nunca & A veces & Muchas veces & Siempre \\
\hline
\end{tabular}

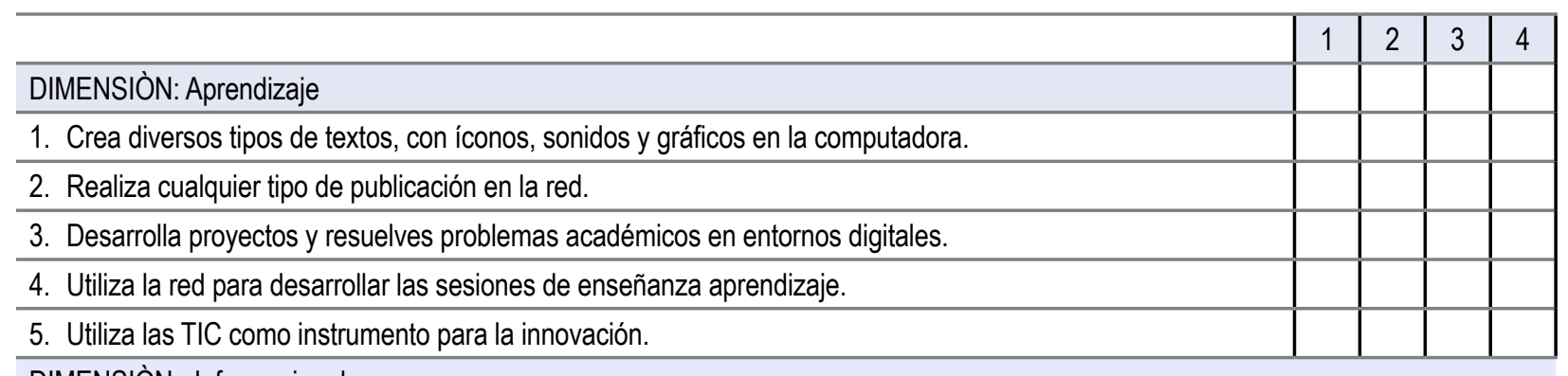

DIMENSIÒN : Informacional

6. Usa sistemas informáticos para acceder a información, recursos y servicios.

7. Utiliza diferentes fuentes de búsqueda según el tipo y el formato de la información: texto, imagen, datos numéricos, mapa, audiovisual y audio

8. Guarda, archiva y recupera la información en Internet.

9. Conoce herramientas y recursos para la buena gestión del conocimiento en ámbitos digitales.

10. Evalúa la utilidad de la información, los recursos y los servicios disponibles

DIMENSIÒN : Comunicativa

11. Se comunica mediante dispositivos digitales.

12. Verifica la calidad y el contenido de la comunicación atendiendo a las necesidades propias y de los demás.

13.Utiliza herramientas de elaboración colectiva de su conocimiento en tareas y proyectos educativos

14.Participa proactivamente en entornos virtuales de aprendizaje, redes sociales y espacios colaborativos.

DIMENSIÒN : Cultura digital

15. Contribuye al aprendizaje mutuo con herramientas digitales.

16. Orienta adecuadamente la identidad digital en Internet.

17. Actúa de forma legal respecto a los derechos de propiedad del software

18. Respeta los diferentes ámbitos de propiedad de los contenidos digitales.

19. Reflexiona sobre la dimensión social y cultural de la sociedad del conocimiento.

20.Propicia el ejercicio responsable de la ciudadanía digital.

DIMENSIÒN : Tecnológica

21. Utiliza con eficacia los dispositivos informáticos propios de las TIC.

22. Utiliza las funciones de navegación en dispositivos informáticos en Internet.

23. Apoya en la configuración del software de la institución educativa.

24. Instala, actualiza y desinstala software o dispositivos informáticos.

25. Cuida de los dispositivos, el software y los contenidos o servicios digitales empleados.

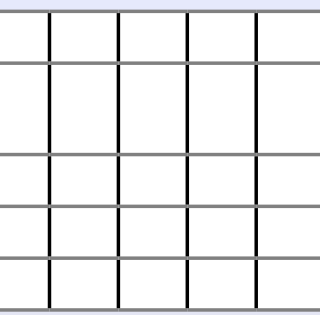




\section{ANeXo 2}

\section{CUESTIONARIO SOBRE DESARROLLO PROFESIONAL}

\section{Instrucciones:}

Estimado docente, la presente encuesta tiene el propósito de recoger información sobre la el desarrollo profesional. Mucho le agradeceré marcar con un aspa "X" en el recuadro que corresponda según su percepción. Esta encuesta tiene el carácter de anónimo, y su procesamiento será reservado, por lo que le solicitamos sinceridad en las respuestas.

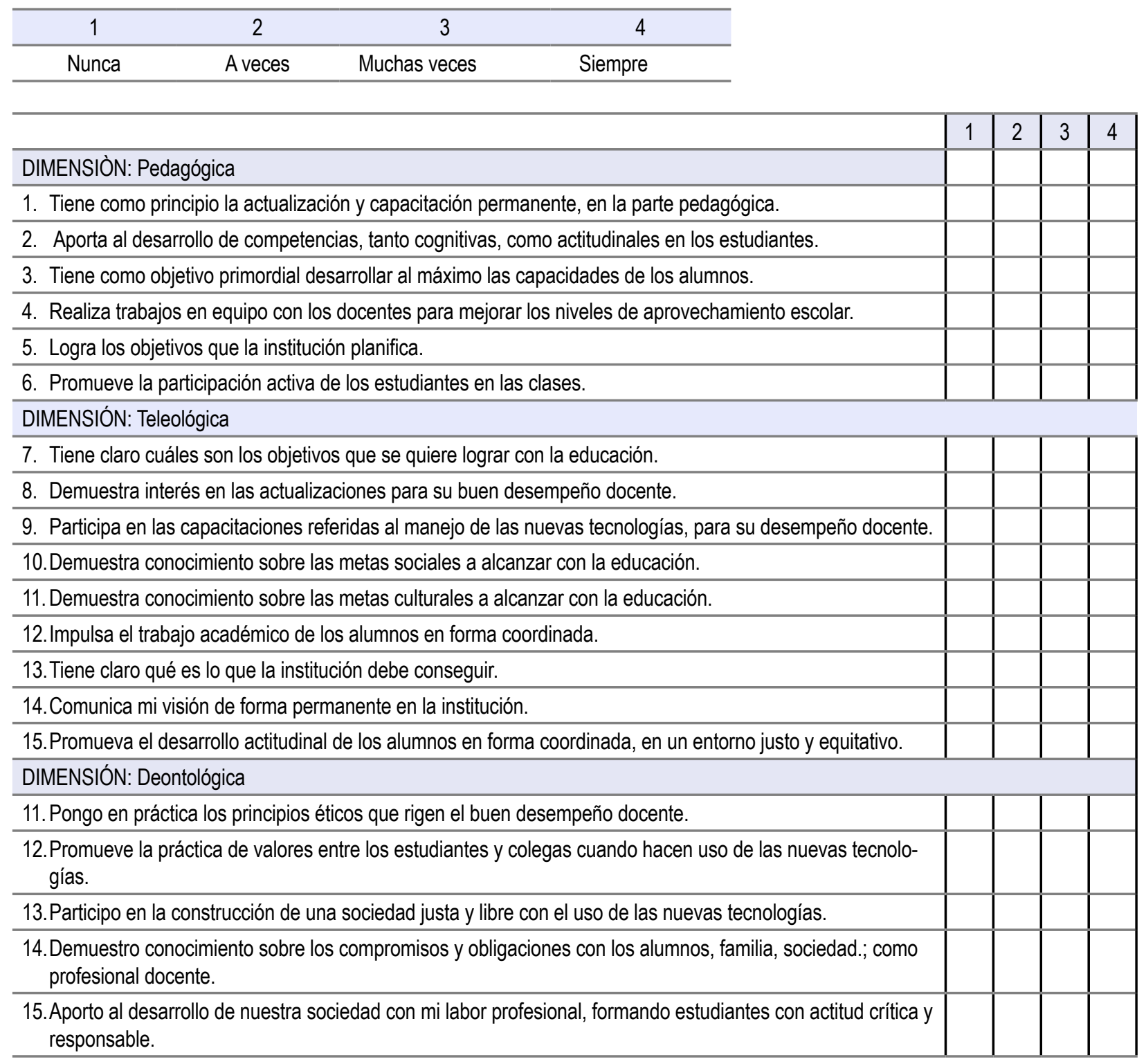


Competencia digital y desarrollo profesional de los docentes de dos instituciones de educación básica regular del distrito de Los Olivos, Lima-Perú

\section{Anexo 3 \\ TÉRMINO DE CONSENTIMIENTO ÉTICO LIBRE Y CLARO}

La investigación se titula "Competencias digitales y el desarrollo profesional docente de EBR", de autoría de un equipo de investigadores de la Universidad César Vallejo, Filial Lima, Campus Lima Norte, teniendo como objetivo central: establecer la relación de las variables de estudio.

Los instrumentos a ser aplicados en el estudio cuantitativo permitirán establecer los niveles alcanzados por los docentes en las dimensiones de las variables estudiadas y establecer la relación entre los datos obtenidos de las variables. La información brindada tiene carácter confidencial garantizado; se le pide poner su nombre en cada instrumento para identificar que ambos pertenecen a la misma persona. Además, responder en forma individual y contestar siempre cada ítem.

La evaluación tiene una duración de media a una hora, durante la evaluación se respeta su rechazo en participar o retirar su consentimiento, dado que su participación es voluntaria.

Lima, 15 de abril del 2018

\begin{tabular}{ll}
\multicolumn{1}{c|}{ Evaluado (o) } & \multicolumn{1}{c}{ Evaluador (a) } \\
Nombre del evaluado (a) & Nombres de Investigadores \\
DNI: & DNI: \\
& \\
& Universidad César Vallejo \\
& Filial Lima Norte
\end{tabular}

